\title{
Erratum to: The carbon dioxide evasion cycle of an intermittent first-order stream: contrasting water-air and soil-air exchange
}

\author{
Arún Looman · Damien T. Maher · Elise Pendall • \\ Adrian Bass • Isaac R. Santos
}

Published online: 29 May 2017

(C) Springer International Publishing Switzerland 2017

\section{Erratum to: Biogeochemistry (2017) 132:87-102 DOI 10.1007/s10533-016-0289-2}

Our paper, published in Biogeochemistry (Vol. 132, 2017, pp. 87-102), contains a calculation error in the determination of the $\mathrm{C}$-gas flux rates. To correct this error all $\mathrm{CO}_{2}$ flux rates should be multiplied by 3.66 , and all $\mathrm{CH}_{4}$ flux rates multiplied by 1.34 . As a result, the sustained global warming potentials of mean daily water-air $\mathrm{CH}_{4}$ contributions to total $\mathrm{C}$-gas emissions from the S1 study segment were 39\% the sustained global warming potentials of mean soil $\mathrm{CO}_{2}$ emissions over the 20-year time frame and $29 \%$ the measured background water-air emissions over the 100-year time frame. The revised Table 2 below reports correct values for the paper. This does not alter any of the statistical analyses, other interpretations, or general conclusions of the manuscript.

The online version of the original article can be found under doi:10.1007/s10533-016-0289-2.

A. Looman $(\bowtie) \cdot$ D. T. Maher · I. R. Santos

National Marine Science Centre, Southern Cross

University, Coffs Harbour, NSW, Australia

e-mail: arun.looman@hotmail.com

\section{A. Looman · D. T. Maher · I. R. Santos}

School of Environment, Science, and Engineering,

Southern Cross University, Lismore, NSW, Australia

\section{E. Pendall · A. Bass}

Hawkesbury Institute for the Environment, Western Sydney University, Richmond, NSW, Australia

\section{A. Bass}

Department of Geographical and Earth Sciences, University of Glasgow, Glasgow, UK 
Table 2 Comparison summary of mean $\mathrm{CO}_{2}$ concentration and fluxes for temporary ponds, perennial flowing rivers and streams, seasonally ephemeral watercourses, lakes, and wetlands worldwide ( $\pm \mathrm{SD}$; ranges shown in parentheses)

\begin{tabular}{|c|c|c|c|c|}
\hline Location/watercourse type & $\begin{array}{l}\text { Flux wet } \\
(\mathrm{mmol} \mathrm{m} \\
\left.{ }^{\mathrm{f}} \mathrm{d}^{-1}\right)\end{array}$ & $\begin{array}{l}\text { Flux wet } \\
(\mathrm{mmol} \mathrm{m} \\
\left.\mathrm{m}^{-2} \mathrm{~d}^{-1}\right)\end{array}$ & $\begin{array}{l}\text { Flux dry } \\
\left(\mathrm{mmol} \mathrm{m} \mathrm{m}^{-2} \mathrm{~d}^{-1}\right)\end{array}$ & References \\
\hline $\begin{array}{l}\text { River Fluvià, Spain/seasonally } \\
\text { ephemeral }\end{array}$ & $\begin{array}{l}79^{*} \\
(41-96)\end{array}$ & $\begin{array}{l}24^{*} \\
(22-41)\end{array}$ & $\begin{array}{l}212^{*} \\
(36-455)\end{array}$ & von Schiller et al. (2014) \\
\hline Iberia, Spain/seasonally ephemeral & $306 \pm 206$ & - & $\begin{array}{l}781 \pm 390 \\
(342-1533)\end{array}$ & Gómez-Gener et al. (2016) \\
\hline Tucson, Arizona/seasonally ephemeral & - & - & $\begin{array}{l}44 \pm 8^{\mathrm{p}, \mathrm{se}, \mathrm{v}} \\
(20-3173)\end{array}$ & Gallo et al. (2013) \\
\hline Worldwide/small ponds $<0.001 \mathrm{~km}^{2}$ & - & $35 \pm 5^{\mathrm{m}, \mathrm{se}}$ & - & Holgerson \& Raymond (2016) \\
\hline Mediterranean/temporary ponds & - & $\begin{array}{l}10^{* \mathrm{v}} \\
(1-70)\end{array}$ & $(7-526)^{* v}$ & Catalán et al. (2014) \\
\hline Worldwide/flowing waters & $766^{\mathrm{m}}$ & - & - & Raymond et al. (2013) \\
\hline Conterminous USA/flowing waters & $\begin{array}{l}541 \pm 182^{\mathrm{m}} \\
(201-914)\end{array}$ & - & - & Butman \& Raymond (2011) \\
\hline Boreal/lakes & - & $33^{\mathrm{m}}$ & - & Weyhenmeyer et al. (2015) \\
\hline Worldwide/lakes and reservoirs & - & $24^{\mathrm{m}}$ & - & Raymond et al. (2013) \\
\hline Tropical/wetlands & - & $\begin{array}{l}107 \pm 50^{v} \\
(20-141)\end{array}$ & $\begin{array}{l}255 \pm 111^{\mathrm{p}, \mathrm{v}} \\
(69-493)\end{array}$ & Sjögersten et al. (2014, Table 4) \\
\hline Boreal/fen ${ }^{\top \oplus}$ & - & $117^{\mathrm{v}}$ & $144^{\mathrm{p}, \mathrm{v}}$ & Sonnentag et al. (2010) \\
\hline This study & $\begin{array}{l}244 \pm 140^{\mathrm{m}, \mathrm{v}} \\
(-34 \text { to } 1278)\end{array}$ & $\begin{array}{l}111 \pm 75^{\mathrm{v}} \\
(0-206)\end{array}$ & $\begin{array}{l}264 \pm 101^{\mathrm{v}} \\
(101-420)\end{array}$ & \\
\hline
\end{tabular}

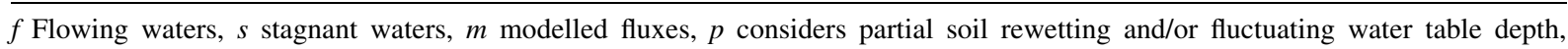
typically $<0 \mathrm{~m}$

* Median values, se \pm standard error, $v$ includes vegetated and/or non-vegetated soils

- Excludes floodplains, ${ }^{\top \oplus}$ Considers fluxes during the snow-free period 